\title{
Target star catalogue for Darwin: Nearby Habitable Star Systems
}

\author{
L.Kaltenegger, ${ }^{12} \dagger$, C.Eiroa ${ }^{23}$, A. Stankov ${ }^{2}$, \\ and M. Fridlund ${ }^{2}$ \\ ${ }^{1}$ Harvard-Smithsonian Center for Astrophysics, 60 Garden St, Cambridge, MA02138, USA \\ email:lkaltenegger@cfa.harvard.edu \\ ${ }^{2}$ European Space Agency ESTEC, P.O.Box 299, NL-2200AG Noordwijk, The Netherlands \\ email:Malcolm.Fridlund@esa.int, astankov@rssd.esa.int \\ ${ }^{3}$ Universidad Autonoma de Madrid, Cantoblanco, 28049 Madrid, Spain \\ email:carlos.eiroa@uam.es
}

\begin{abstract}
In order to evaluate and develop mission concepts for a search for Terrestrial Exoplanets, the European Space Agency has prepared a list of potential target systems. In this paper we present the criteria for selecting potential target stars suitable for the search for Earth like planets. We also establish the habitable zone for the stellar systems. Planets found within these zones would be potentially able to host complex life forms. The Darwin target star catalogue was created from the Hipparcos catalogue by examining the information on distance, spectral classification, multiplicity and stellar variability. Additional information on the targets stars including spectral type, metallicity, X-ray luminosity, rotation and Strömgren photometry from several other catalogues are included to determine the target star sample. Design constraints of the mission are added to derive a final target star catalogue of 262 target stars excluding and 447 target stars including M stars for the Darwin mission. We also discuss the completeness of sample for different classes of stars.
\end{abstract}

Keywords. extrasolar planets, interferometers, catalogs, planetary systems, Earth, planets

\section{Introduction}

Among current scientific endeavours, the question if life exists only the Earth or elsewhere in the Universe is arguably one of the most important. Many issues remain to be solved before the "big question" of life in the Universe as contrasted to life in our Solar System can be addressed. One important constraint is: what and where is the sample of stars around which we find planets suitable for life? To model realistic observation scenarios the luminosity, distance and radius of the star and the corresponding habitable zone (HZ) for an Earth-like planet has to be taken into account. The optimization and success of the mission depends critically on those parameters. Analysis of the planetary light requires that the stellar light is suppressed to a high degree. This is done in the IR by a technique called nulling interferometry. Briefly, this implies that achromatic phase shifts are applied to the beams collected by individual telescopes before recombination such that the on-axis light, i.e. the stellar light, is cancelled by destructive interference, while the much weaker planetary light emitted at a certain small, off axis angle interferes constructively. In the visible wavelength range interferometry is currently impractical, NASA's Terrestrial Planet Finder (TPF) concept in the visible is implemented as a very large coronagraph. Detailed simulations critically depend on correct data on the target stars.

$\dagger$ Present address: CfA, 60 Garden st, MS20, Cambridge, MA02138, USA 


\section{Target star catalogue}

The sample of nearby stars is limited. As a preparatory activity for the future missions, as well as the required ground based programs, both NASA and ESA are preparing input catalogues. It is expected that consortia will form across the whole world, which will start determining the properties of the target stars. In preparation for the Darwin mission a catalogue of stellar systems that are potentially candidates has been prepared (see Kaltenegger et al. 2005, Eiroa et al. 2003, Kaltenegger 2004). A preliminary list of target stars from the Gliese star catalogue prepared by Ollivier and Leger had been used earlier for calculations for the Darwin feasibility study.

The Darwin target catalogue is based on the Hipparcos catalogue. Using various existing data archives we established a catalogue of well characterized stars that will be used as the Darwin target star list (see Kaltenegger et al. 2005). We use distance, spectral classification, multiplicity and stellar variability and adding information including spectral type, metallicity, X-ray luminosity, rotation as well as Strömgren photometry from several other catalogues to establis a preliminary target star sample. For many of the nearby late $\mathrm{K}$ and $\mathrm{M}$ stars data on multiplicity, rotation, metallicity etc are still missing, thus modifications of the Darwin catalogue will be implemented as such data becomes available. Further developing this sample, and taking several different configurations of the Darwin interferometer into account we have now a final sample, extending out to a distance of 25pc from Earth. As what concerns F, G and early K-type main sequence stars it is considered complete. Later stellar types, which should be numerous, are conspicuous in their absence. This is not expected to impact the Darwin or TPF missions, since ESA's GAIA mission will fly in 2012 and it is expected to provide parallaxes down to 20 microarcseconds for objects brighter than R-magnitude 22 within $1 \frac{1}{2}$ years, thus completing our knowledge of nearby stars out to at least $25 \mathrm{pc}$ and for essentially all of class M.

Four selection criteria were adopted to first generate the "Darwin all sky target catalogue" that does not take the instrument design into account and assumes access to the whole sky. An additional instrument design criterion was added v) based on the instrument design leading to the "Darwin prime target star catalogue": i) FGKM stars within a distance of $25 \mathrm{pc}$, ii) main sequence stars nature based on the $B-V$ index: $(0.25<B-V<1.93)$ and their assigned luminosity class iii) stars with magnitudes smaller than 12 in $\mathrm{V}$, iv) single stars, v) stars within a cone of aperture \pm 45 degree of the ecliptic plane.

The Darwin all sky target catalogue consists of 628 including and 387 stars excluding M stars. The current analysis results in a Darwin prime target star list of 447 target stars including and 262 excluding $M$ stars consistent with the selection criteria within 25 pc including instrument limitations see figure 1 and figure 2 .

From the color magnitude diagram shown in figure 1, it is obvious that most target stars are still on the main sequence while a few have already evolved off it. The different colors show the different stellar types.

The interferometric systems suggested for Darwin and TPF-I operate in the mid-IR, the coronagraph suggested for TPF-C in the visible. For the former it is thus the thermal emission emanating from the planet that is detected and analysed while for the latter the reflected stellar flux is measured. This means if you want to observe earth-like planets in the HZ around a given star, the flux in the IR will to first order be constant for a given planetary size. The suppression of the primary's light will, on the other hand, be progressively easier for later spectral types. Surprisingly enough, it may thus be easier to detect a habitable Earth around an $\mathrm{M}$ dwarf than around something more akin to our 

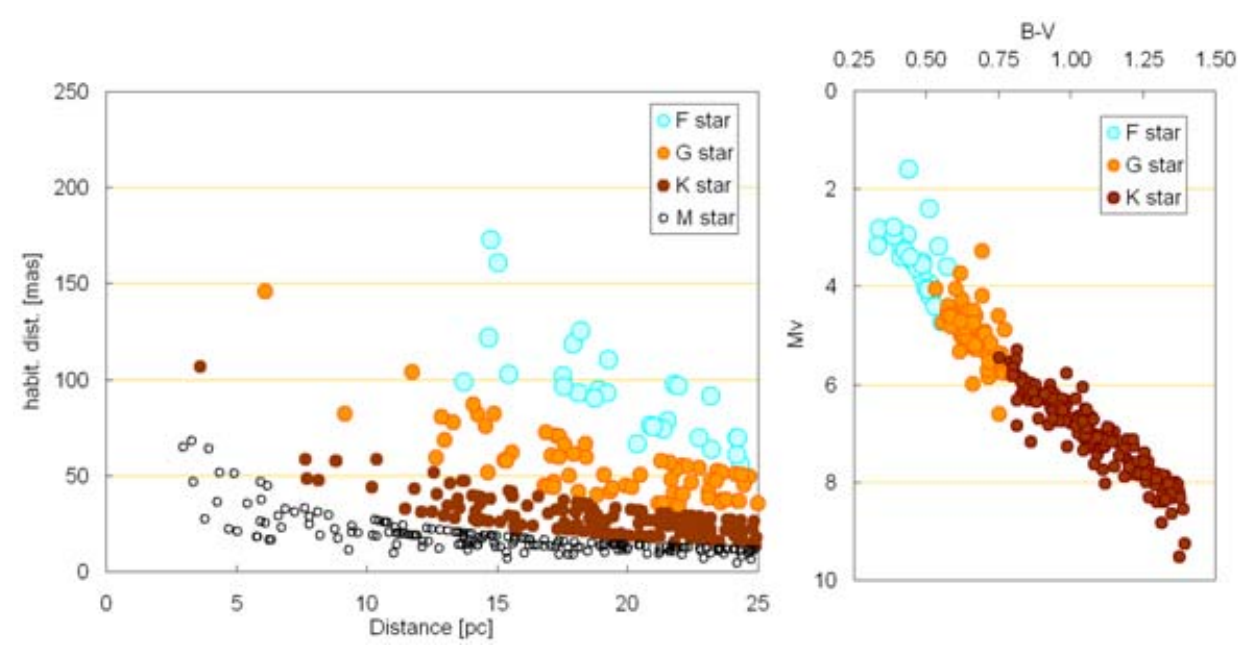

Figure 1. Distance of the HZ in mas (left) Color-magnitude diagram for the Darwin prime target stars (right).
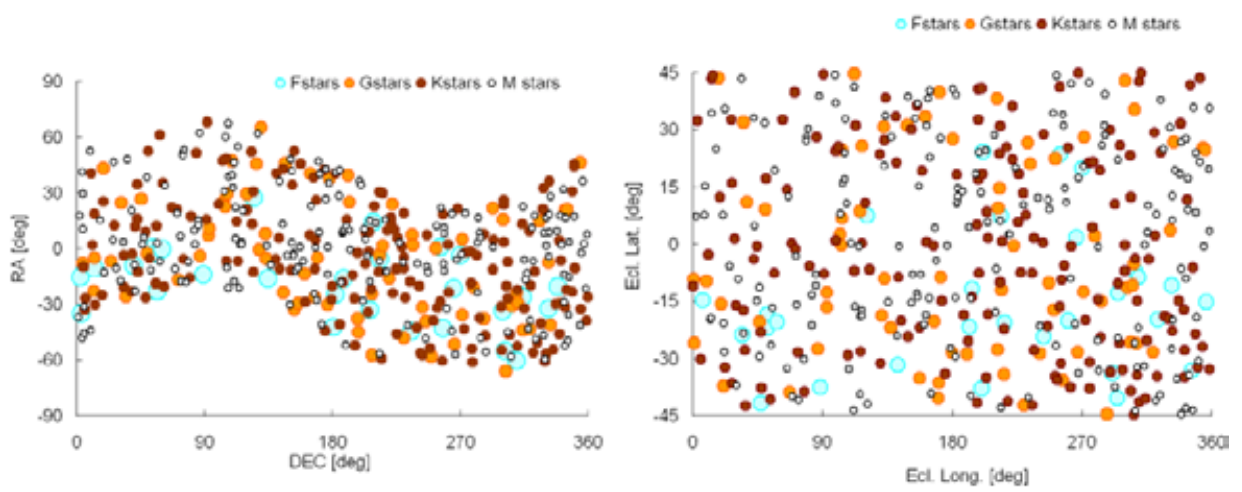

Figure 2. Position for the 45-degree cone Darwin target stars in RA, DEC (left) and ecliptic coordinates(right).

own Sun. The HZ moves closer to the star for later stellar systems thus the interferometric systems has to be adapted to each individual target system for optimum performance, implemented as a free flyer array. The baseline of the interferometers has to increase to resolve $\mathrm{M}$ star planetary-systems at larger distances, a constraint that is taken into account for the $\mathrm{M}$ target systems at largest distance in the target star catalogue.

The target star list presented is used for the trade off studies of different mission architectures for the proposed Darwin mission (see Kaltenegger \& Karlsson (2004), Hartog, Absil, Kaltenegger et al. (2004) for the TPF-I architecture see Dubovitsky \& Lay (2004)). Limitations imposed by the current instrument design are e.g. the 45-degree observation cone in anti-sun direction, a minimum distance to a close binary companion that cannot be simultaneously nulled while searching for a planet and a minimum observation time of the star in the field of view of the instrument. The 45-degree cone in the selection criteria is given by constraints of the sunshields on each free flyer resulting in a sky-coverage of $70.7 \%$. A new concept is under investigation that would allow the instrument to cover $95 \%$ of the whole sky. Considering this we included statistics for an all sky survey as 
well as for the Darwin 45-degree cone concept. The limitation to a \pm 45 -degree cone in anti-sun direction places no severe constraint on the mission or limits its performance.

\subsection{Completeness of the catalogue}

From the catalogues, some important conclusions can be immediately drawn. Within 25pc, we find about 700 single stars, with well defined Hipparcos distances (see Kaltenegger et al. 2005). Of these about half are F, G and K type objects, while the remainder consists exclusively of $\mathrm{M}$ dwarfs. The $\mathrm{F}$ and $\mathrm{G}$ type stars known within a distance of $25 \mathrm{pc}$ can be considered to be a complete sample. The same is not true for the later spectral types, especially for the M-dwarfs. As one progresses more than about 10pc from the Earth, the sample of known M-dwarfs dwindle rapidly. The incompleteness is easily seen in that between 10 and $20 \mathrm{pc}$ we find $14 \mathrm{~F}, 35 \mathrm{G}, 87 \mathrm{~K}$ and $140 \mathrm{M}$ single stars in the Darwin prime target star sample, while for the volume between 20 and 25pc the corresponding numbers are 15, 34, 77 and 45. In the all sky target list, the lack in M stars at further distance can be seen even more severely. Naturally G stars will turn out to be prime targets as they are similar to our sun and the likelihood that under similar conditions, planets with similar life-forms showing similar atmospheric features evolve, seems high. Including other stellar types provides the unique opportunity to characterize planets around different host stars. Extrasolar Giant Planets have been found around different stellar types thus the target sample should reflect those stellar classes. It is essential that we have a certain number of targets stars to derive conclusions for detections or non-detections. Even so the frequency of extra-solar terrestrial planets can only be guessed. We believe that a sample has to consist of a minimum of 100 to 150 stars to be able to derive conclusions.

\section{Conclusions}

In preparation of the Darwin mission we established a catalogue of stellar systems of nearby stars that are potentially habitable to complex life. The list was created from the Hipparcos catalogue by examining the information on distance, stellar variability, multiplicity, location and spectral classification. The Darwin all sky target catalogue consists of 628 target stars including and 387 target stars excluding M stars, reflecting an alternative mission concept that would allow access to the whole sky. Design constraints of the current architecture of the Darwin mission limits the sky access to \pm 45 -degree in ant-isun direction. Applying these design constraints we derive a prime Darwin target star sample of 262 target stars excluding and 447 including $\mathrm{M}$ stars within 25 pc as potential targets for the search for earth-like planets.

\section{References}

Dubovitsky, S. \& Lay, O.P. 2004, SPIE 5491

Eiroa, C., Fridlund, M., \& Kaltenegger, L. 2003, ESA-SP 539

Hartog, R., Absil, O, Kaltenegger, L., Karlsson, A., \& DArcio, L. 2004, SPIE 5491

Kaltenegger, L. 2004, PhD thesis, Karl-Franzens University Graz, Austria

Kaltenegger, L \& Karlsson A. 2004, SPIE 5491

Kaltenegger, L, Eiroa, C., Stankov, A., \& Fridlund, M. 2005, in prep

Karlsson, A.L., Wallner, O., \& Perdigues Armengol, J. M 2004 SPIE 5491

Kasting, J.F., Whitmire, D.P., \& Reynolds, R.T. 1993 Icarus 101,108 